ESTUDOS EM ARQUEOMETRIA E ARTE POR METODOLOGIAS NUCLEARATÔMICO-MOLECULARES NÃO DESTRUTIVAS NO LABORATÓRIO DE FÍSICA NUCLEAR APLICADA DA UNIVERSIDADE ESTADUAL DE LONDRINA

STUDIES IN ARCHEOMETRY AND ART BY NON-DESTRUCTIVE NUCLEARATOMIC-MOLECULAR METHODOLOGIES IN THE APPLIED NUCLEAR PHYSICS LABORATORY OF THE STATE UNIVERSITY OF LONDRINA

Carlos Roberto Appoloni

Como citar este artigo:

APPOLONI, Carlos Roberto. Estudos em arqueometria e arte por metodologias nuclear-atômico-moleculares não destrutivas no laboratório de física nuclear aplicada da Universidade Estadual de Londrina. In: Cadernos do Lepaarq, v. XV, n.30., p. 219-228, Jul-Dez. 2018. 


\title{
Estudos em arqueometria e arte por metodologias nuclear-atômico-moleculares não destrutivas no laboratório de física nuclear aplicada da Universidade Estadual de Londrina
}

\author{
Carlos Roberto Appoloni ${ }^{a}$
}

\begin{abstract}
Resumo: No Laboratório de Física Nuclear Aplicada (LFNA - DFIS/UEL) diferentes combinações de metodologias nucleares, atômicas e moleculares têm sido empregadas para estudos de objetos do patrimônio cultural. Metodologias como a Fluorescência de Raios $\mathrm{X}$ (nas diferentes modalidades EDXRF, PXRF, TXRF), a Espectroscopia Raman e a Transmissão de Raios Gama têm sido empregadas no estudo de diferentes tipos de objetos arqueológicos e, quando necessário, em colaboração com outros laboratórios, em conjunto com técnicas como a Emissão de raios X induzida por partículas (PIXE), Espectroscopia Mössbauer, Microscopia Eletrônica, Radiografia Digital, Difração de Raios X e Retroespalhamento Rutherford (RBS), entre outras. Neste contexto, apresenta-se uma síntese dos trabalhos realizados desde o início do envolvimento do LFNA com Arqueometria e Artes, fazendo um pequeno recorte dentro da larga gama de objetos do patrimônio cultural analisados, focando alguns materiais cerâmicos.
\end{abstract}

\section{Palavras Chave:}

Arqueometria; Métodos; Nucleares; Atômicos; Moleculares.

\begin{abstract}
Different combinations of nuclear, atomic and molecular methodologies are employed In the Laboratory of Applied Nuclear Physics (LFNA - DFIS / UEL) to study objects of cultural heritage. Methodologies such as X-Ray Fluorescence (in the different modalities EDXRF, PXRF, TXRF), Raman Spectroscopy and Gamma Ray Transmission have been employed to the study of different types of archaeological objects and when necessary, in collaboration with other laboratories, in conjunction with techniques such as particle induced X-ray emission (PIXE), Mössbauer Spectroscopy, Electron Microscopy, Digital Radiography, X-ray Diffraction and Rutherford Backscatter (RBS), among others. In this context, a synthesis of work accomplished since the beginning of the LFNA's involvement with Archaeometry and Arts is presented, making a small cut within the wide range of cultural heritage objects analyzed, focusing on some ceramic materials.
\end{abstract}

\section{Keywords:}

Archaeometry; Methods; Nuclear; Atomic; Molecular.

$\boldsymbol{a}$ Universidade Estadual de Londrina (UEL), Brasil; Professor Doutor, Programa de Pós-Graduação em Física, Conselho Nacional de Desenvolvimento Científico e Tecnológico (CNPq), Brasil; Proc. No. 305.695/2013-4 e INCT-FNA CNPq Proc. No. 464898/2014-5. E-mail: appoloni@uel.br 


\section{INTRODUÇÃO}

O Laboratório de Física Nuclear Aplicada (LFNA) da Universidade Estadual de Londrina (UEL) tem se envolvido desde 1993 com uma grande variedade de questões a serem respondidas sobre objetos do Patrimônio Cultural, com materiais de diferentes geometrias e matrizes, o que implica em uma grande variedade de dificuldades experimentais para enfrentar e resolver (APPOLONI, 2013; APPOLONI et al., 2013a). As cerâmicas arqueológicas têm sido os objetos de estudo mais freqüentes desde o início (APPOLONI, et al., 1994; APPOLONI, 1996; APPOLONI et al.,1997) e até o presente ( MILHEIRA et al, 2009; IKEOKA et al. 2012; IKEOKA, 2014; IKEOKA et al. 2017; JUSSIANI et al. 2017), mas outros materiais tem sido também estudados, como imagens policromáticas em madeira (BLONSKI e APPOLONI, 2014), pinturas murais (RIZZO et al, 2007), moedas (PARREIRA et al., 2009), obsidianas (GALVÃO et al., 2010), arte rupestre (APPOLONI et al. 2010; APPOLONI et al. 2014), e pinturas de cavalete (APPOLONI et al., 2007; RAVAUD et al., 2009 e APPOLONI et al. 2013b), entre muitos outros tipos diferentes de objetos. Será apresentada a revisão de alguns trabalhos realizados desde o início do envolvimento do LFNA com Arqueometria, realizando um pequeno recorte focando materiais cerâmicos, dentro da larga gama de objetos do patrimônio cultural analisados.

\section{DENSITOMETRIA - TRANSMISSÃO DE RAIOS GAMA E MICROTOMOGRAFIA}

A metodologia de densitometria por transmissão de raios gama consiste em se fazer uma "varredura" da amostra, para se inspecionar a estrutura interna e homogeneidade da pasta cerâmica, através da atenuação da radiação gama pelos diferentes fragmentos. Várias fontes radioativas podem ser usadas, em função da escolha da energia dos raios gama ser feita levando em conta a espessura das amostras. A fonte tipicamente usada é a de ${ }^{241} \mathrm{Am}$, que se aplica bem para o intervalo usual de espessura e densidade das cerâmicas arqueológicas.

O primeiro trabalho publicado por membros do LFNA reportando resultados de densitometria com raios gama (APPOLONI, et al., 1994), tratou de nove fragmentos de cerâmicas da tradição Caingangue pertencentes à coleção arqueológica do Museu Histórico Padre Carlos Weiss, da Universidade Estadual de Londrina (UEL). Neste trabalho foram usadas duas fontes de raios gama, ${ }^{241} \mathrm{Am}$ (raios gama de 59,5 keV) e ${ }^{153} \mathrm{Gd}$ (raios gama de 44,0 e 99,8 keV), cadeia eletrônica padrão de espectrometria gama, um detector cintilador de $\mathrm{NaI}(\mathrm{Tl})$ de 2", sistema para movimentação milímétrica vertical e horizontal das amostras e colimadores de $\mathrm{Pb}$ na saída das fontes radioativas e entrada do detector. As gamagrafias em passos de $1 \mathrm{~mm}$ ao longo das amostras em três posições diferentes, mostraram considerável diferença de densidade e composição entre os fragmentos. O número atômico médio da composição do fragmento mais denso era $36 \%$ maior que o do fragmento menos denso do conjunto de amostras. Por outro lado, o valor da densidade ao longo de cada amostra mostrou que alguns fragmentos eram muito homogêneos, enquanto que outros ou eram muito heterogêneos ou apresentavam a presença de chamotes de densidade bem mais alta que a pasta cerâmica.

Dentre outros trabalhos do LFNA empregando esta metodologia, destacamos o trabalho de SILVA, et al. 2004. Este trabalho trata da análise arqueométrica de diferentes conjuntos cerâmicos arqueológicos e etnográficos atribuídos a populações Tupi. O objetivo foi ressaltar a importância das análises arqueométricas para o entendimento dos materiais cerâmicos. Estas análises são um meio privilegiado de acesso a um conjunto de dados fundamentais para a compreensão dos processos tecnológicos de diferentes populações, que permite aos arqueólogos melhor compreenderem as características de performance dos artefatos cerâmicos, possibilitando a construção de tipologias mais refinadas e, por outro lado, ampliando o entendimento sobre as características das tradições arqueológicas. Um dos métodos empregados foi o de densitometria por transmissão de Raios Gama, cujo objetivo foi identificar a existência ou não de uso de 
antiplásticos na composição da pasta cerâmica e perceber as possíveis diferenciações no processo de preparação da argila e construção do vasilhame cerâmico.

As amostras foram examinadas com passo de translação horizontal de $1 \mathrm{~mm}$, em três diferentes níveis verticais, espaçados de $6,5 \mathrm{~mm}$, em região afastada das bordas.

O estudo arqueométrico foi realizado com fragmentos cerâmicos arqueológicos e etnográficos oriundos de duas regiões geográficas distintas: da região amazônica (centro-sul do Estado do Pará) e da região sul (norte do estado do Paraná). Os fragmentos cerâmicos etnográficos foram produzidos pelos Asurini do Xingu e os fragmentos cerâmicos arqueológicos amazônicos foram coletados na área de ocupação dos Asurini do Xingu e na área dos Kayapó-Xikrin do Cateté, sendo identificados como de origem Tupi. Os fragmentos cerâmicos arqueológicos da região sul foram coletados na Fazenda Santa Dalmácia, no município de Cambe, norte do Paraná, e foram identificados como pertencentes à "Tradição Tupiguarani”. Diferentes qualidades tecnológicas em termos da densidade e homogeneidade da pasta foram observadas, assim como a presença de vários tipos de anti-plásticos.

Informações sobre a densidade e homogeneidade da pasta, a presença de temperos ou buracos e a porosidade da pasta cerâmica, também podem ser obtidos pela técnica de radiografia digital (CURADO, J. F. et al, 2013; IKEOKA, 2014) ou por microtomografia com raios X (KULKOVA, M. A. e KULKOV, A. M., 2014,).

Mais recentemente passou-se a realizar este tipo de medida de forma mais completa no LFNA através da microtomografia com raios X, com a qual pode-se obter um perfil de distribuição dos tamanhos de poro da amostra, o perfil de distribuição dos tamanhos dos anti-plásticos e a porosidade ao longo da amostra, além da visualização tridimensional completa da mesma (JUSSIANI et al, 2017).

\section{COMPOSIÇÃO QUÍMICA ELEMENTAR - FLUORESCÊNCIA DE RAIOS X}

A técnica de Fluorescência de Raios X por Dispersão em Energia (EDXRF) tem sido amplamente usada no estudo de objetos arqueológicos desde o final dos anos cinqüenta do século XX (HALL et al, 1964) e consiste na excitação dos átomos da amostra por um feixe de raios X, seguida da emissão dos raios X característicos pelos elementos constituintes da amostra e sua detecção (VAN GRIEKEN e MARKOWICZ, 2002).

O primeiro trabalho publicado por membros do LFNA na análise de cerâmicas arqueológicas combinando várias metodologias (APPOLONI et al.,1997) foi um estudo envolvendo Transmissão de Raios Gama, Retroespalhamento Rutherford (RBS) e EDXRF da coleção de fragmentos cerâmicas da tradição Caingangue, pertencentes à coleção arqueológica do Museu Histórico Padre Carlos Weiss, referidos anteriormente. As determinações quantitativas de elementos por RBS e EDXRF permitiram medir a composição não somente da pasta cerâmica, mas também das superfícies externas e internas, permitindo hipotizar sobre a possível composição dos pigmentos ou materiais usados para a decoração plástica. É citada na literatura a difículdade de distinguir visualmente entre, por exemplo, a tinta de hematita aguada e uma tinta orgânica ou uma resina aplicada após a queima (MILLER JR., 1978). A análise multielementar apresentada resolve esta questão. A presença de Fe em altas concentrações nas superfícies interna ou externa em relação à pasta cerâmica é forte indicador do uso de pigmento mineral. No estado do Paraná a hematita não aparece em grandes massas, mas como componente da Laterita, uma crosta de Fe, Alumina e outros elementos, que, após sua formação e endurecimento, aparece na superfície por processos de erosão. É este composto que era usado para a decoração plástica, fazendo com que a análise da composição dos pigmentos mostre $\mathrm{Fe}, \mathrm{Al}$ e elementos-traço característicos de cada região, devido ao complexo processo físico-químico da formação e endurecimento das crostas lateríticas.

A técnica de EDXRF, nas suas modalidades de bancada (EDX), portátil (PXRF) e de reflexão total (TXRF), tem sido utilizada em vários trabalhos de Arqueometria no LFNA e em colaboração com outros laboratórios. 
Um estudo envolvendo o LFNA e o LIN/CENA/USP (CUNHA e SILVA et al., 2005), combinando a EDXRF convencional, a microfluorescência de raios X ( $\mu$-XRF) com fonte de luz Síncrotron e a Microscopia Eletrônica de Varredura acoplada com Fluorescência de Raios X Dispersiva (SEM/EDS), forneceu resultados ainda mais aprofundados para trinta amostras cerâmicas do centro-sul do Estado do Pará (Xikrin e Assurini) e do norte do estado do Paraná (Tupi Guarani), parte delas discutidas nos trabalhos relatados anteriormente. Foi realizado o mapeamento 2D por $\mu$-XRF dos elementos $\mathrm{K}, \mathrm{Ca}, \mathrm{Ti}, \mathrm{Cr}, \mathrm{Mn}, \mathrm{Fe}, \mathrm{Ni}, \mathrm{Cu}, \mathrm{Zn}$ e Rb nas amostras com passo de 20 micra, numa área de 0,20 por $0,36 \mathrm{~mm}$ na pasta cerâmica. A Figura 1 apresenta uma foto das amostras. A Figura 2 apresenta a distribuição 2D para os elementos $\mathrm{Ca}$ e Fe numa das amostras. Com os valores das concentrações medidas por EDXRF realizou-se uma análise multivariada dos dados (HCA com o programa estatístico SAS), obtendo-se o dendrograma mostrado na Figura 3, no qual são observados dois grupos: 1 (com dois sub grupos 1A e 1B), e 2 (com dois sub grupos 2A e 2B).

\section{COMPOSIÇÃO MOLECULAR POR ESPECTROSCOPIA RAMAN}

A base da técnica da espectroscopia Raman é a excitação da amostra por um laser e a detecção do espectro dos deslocamentos Raman observados (diferença entre o número de onda da luz do espalhamento Rayleigh e da luz do espalhamento Raman Stokes). Com este tipo de espectro é possível determinar a composição molecular da amostra. Cada pico corresponde a um modo vibracional excitado de uma molécula presente na amostra, e muitas vezes uma única molécula é capaz de gerar diversos picos. Assim, os picos do espectro caracterizam a composição molecular da amostra (FERRARO et al., 2003; APPOLONI et al., 2014). Desta forma, esta metodologia pode ser empregada tanto para caracterizar os compostos de um pigmento da decoração plástica de uma cerâmica, como a composição mineralógica da pasta e, através desta, inferir a dureza do material, assim como a atmosfera e temperatura de queima do objeto.

Num trabalho do LFNA com fragmentos cerâmicos de sítios arqueológicos localizados no Maranhão (IKEOKA, 2014; IKEOKA et al. 2017), em que foram empregadas seis metodologias de análise (EDXRF, PIXE, Espectroscopia Mössbauer, Espectroscopia Raman, Difração de Raios X e Radiografia Digital), os resultados de espectroscopia Raman (ER) foram relevantes e congruentes com os de outras metodologias (Espectroscopia Mössbauer e Difração de Raios X) para a determinação da atmosfera e temperatura de queima. As amostras analisadas por ER foram provenientes dos seguintes sítios arqueológicos: Bacanga, em São Luis (6 amostras), Panaquatira, em São José de Ribamar (5 amostras) e Rabo de Porco, em Bacabeira (2 amostras). Utilizou-se um espectrômetro Raman portátil, modelo InspectoR da DeltaNu, com laser em $785 \mathrm{~nm}$ com potência máxima de $120 \mathrm{~mW}$, com $8 \mathrm{~cm}^{-1}$ de resolução e alcance espectral de 200-2000 $\mathrm{cm}^{-1}$. Primeiramente, só foram levados em consideração para a análise as bandas cujas larguras à meia altura eram maior que $16 \mathrm{~cm}^{-1}$. Em seguida, as bandas cujas contagens eram menores que 5\% da contagem da banda mais intensa observada também foram descartadas. Por fim, as bandas medidas foram classificadas de acordo com suas intensidades usando a seguinte convenção:

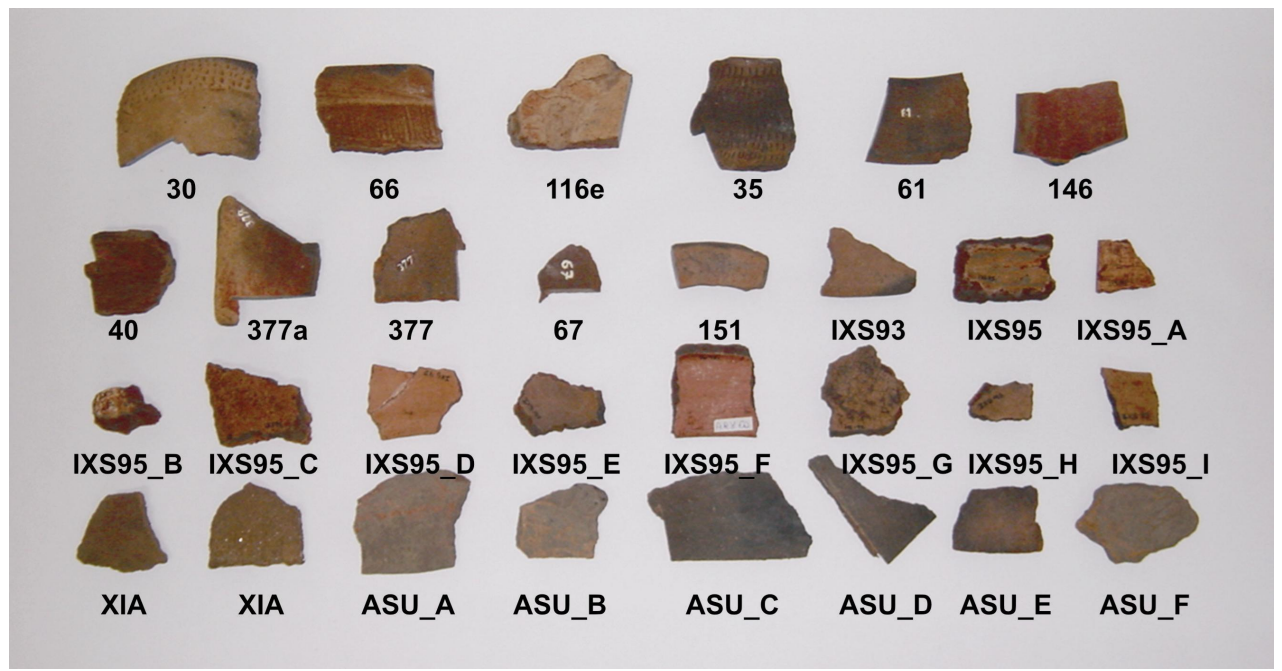

Figura 1 - Fotografia dos fragmentos cerâmicos arqueológicos analisados e seus códigos (extraída de CUNHA e SILVA et al., 2005). 
Muito intenso (mi): 100 a 70\% do maior pico; Intenso (i): 70 a 50\% do maior pico; Médio (m): 50 a 15\% do maior pico; Fraco (f): 15 a 5\% do maior pico. Vamos apresentar apenas alguns dos resultados decorrentes das análises dos espectros medidos. Nos 13 fragmentos analisados por espectroscopia Raman foram detectados hematita, quartzo, wollastonita e albita. As bandas detectadas nas amostras em torno de 263, 574 e $1315 \mathrm{~cm}^{-1}$ indicam assim a presença de hematita nos fragmentos. A presença de hematita indica que a atmosfera de queima da peça cerâmica foi oxidante (OLIVARES et al., 2010). Foram encontradas em todos os fragmentos bandas em 438 (m), 1080 (f) e 1159 (f) $\mathrm{cm}^{-1}$ que correspondem às bandas do quartzo. É sabido que o quartzo é um mineral comum em cerâmicas antigas e sua presença indica que a matéria prima utilizada na fabricação das cerâmicas era rica em sílica. A presença de wollastonita foi indicada devido às bandas detectadas em todas as amostras em torno de 970 (m) e 1080 (f) $\mathrm{cm}^{-1}$. A wollastonita é um dos polimorfos do CaSiO${ }_{3}$, e é uma fase formada na interface entre minerais carbônicos e quartzo a temperaturas acima de $950{ }^{\circ} \mathrm{C}$ (OLIVARES et al., 2010). Isso indica que a temperatura de queima, em algum momento, ultrapassou ou chegou perto dessa temperatura para que houvesse a formação da wollastonita. Outro mineral detectado em todos os fragmentos foi a albita. As bandas do espectro Raman medidas em 492,574 e 1080 $\mathrm{cm}^{-1}$ correspondem às três bandas características da albita. A albita é um mineral que pertence ao grupo de feldspatos plagioclásios e sua presença indica que a temperatura de queima foi abaixo de $950{ }^{\circ} \mathrm{C}$ (RASKOVSKA et al., 2010). A detecção simultânea de albita e da wollastonita nos permite determinar, dentro de certo intervalo, a temperatura limite de queima dos fragmentos cerâmicos analisados. Podemos afirmar que a temperatura máxima de queima não ultrapassou $1.000{ }^{\circ} \mathrm{C}$, caso contrário a albita não seria detectada, ou estaria presente em muito menor intensidade, e deve ter sido maior que $900{ }^{\circ} \mathrm{C}$ para permitir que a wollastonita tenha sido formada e detectada. Portanto, a temperatura de queima deve ter ocorrido no intervalo de $900-1000^{\circ} \mathrm{C}$ e a atmosfera predominante foi oxidante.
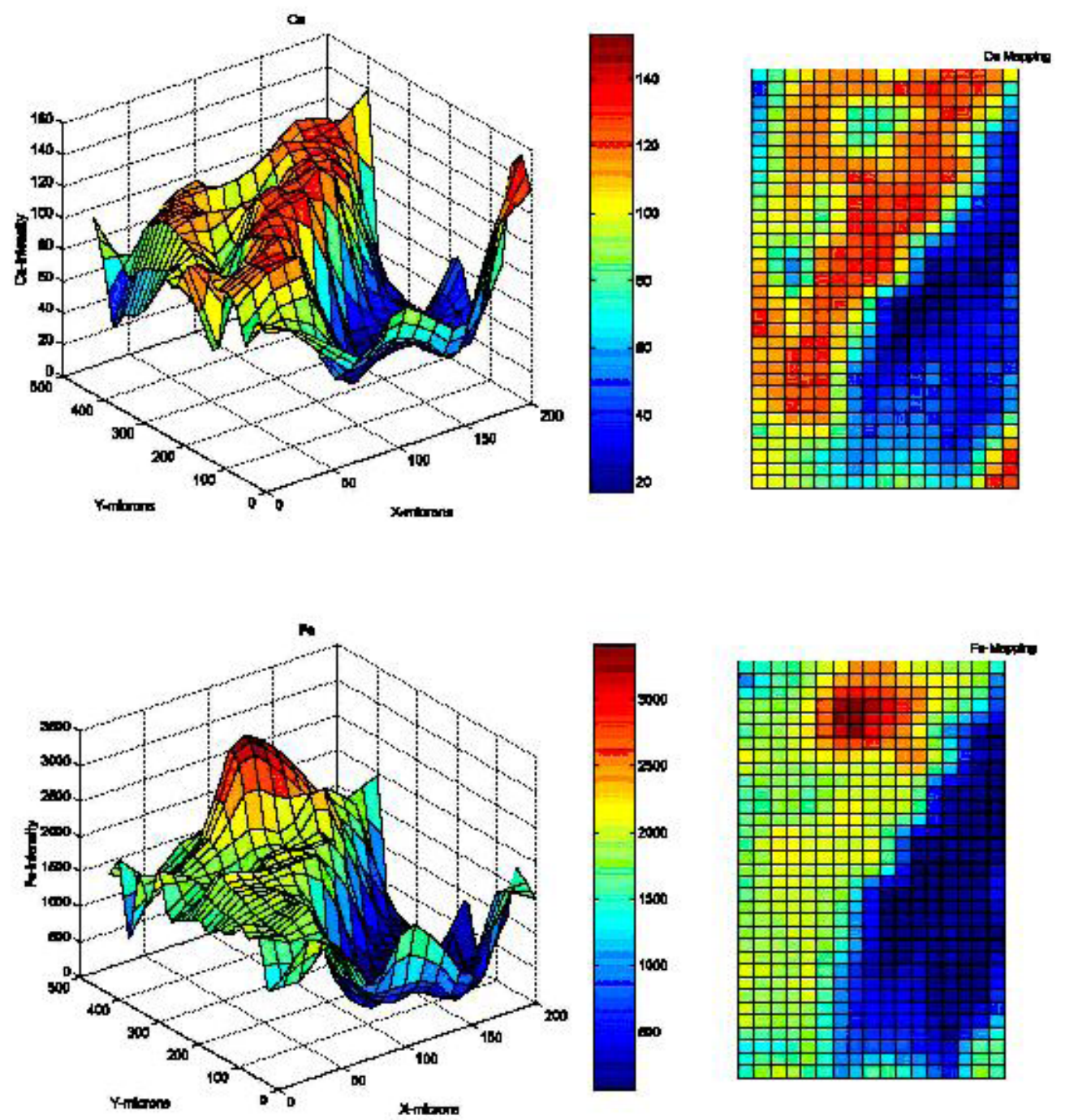

Figura 2 - Mapeamento dos elementos químicos Ca e Fe na pasta da amostra 35 (escala em ppm), extraída de CUNHA e SILVA et al., 2005. Extraída de CUNHA e SILVA et al., 2005. 


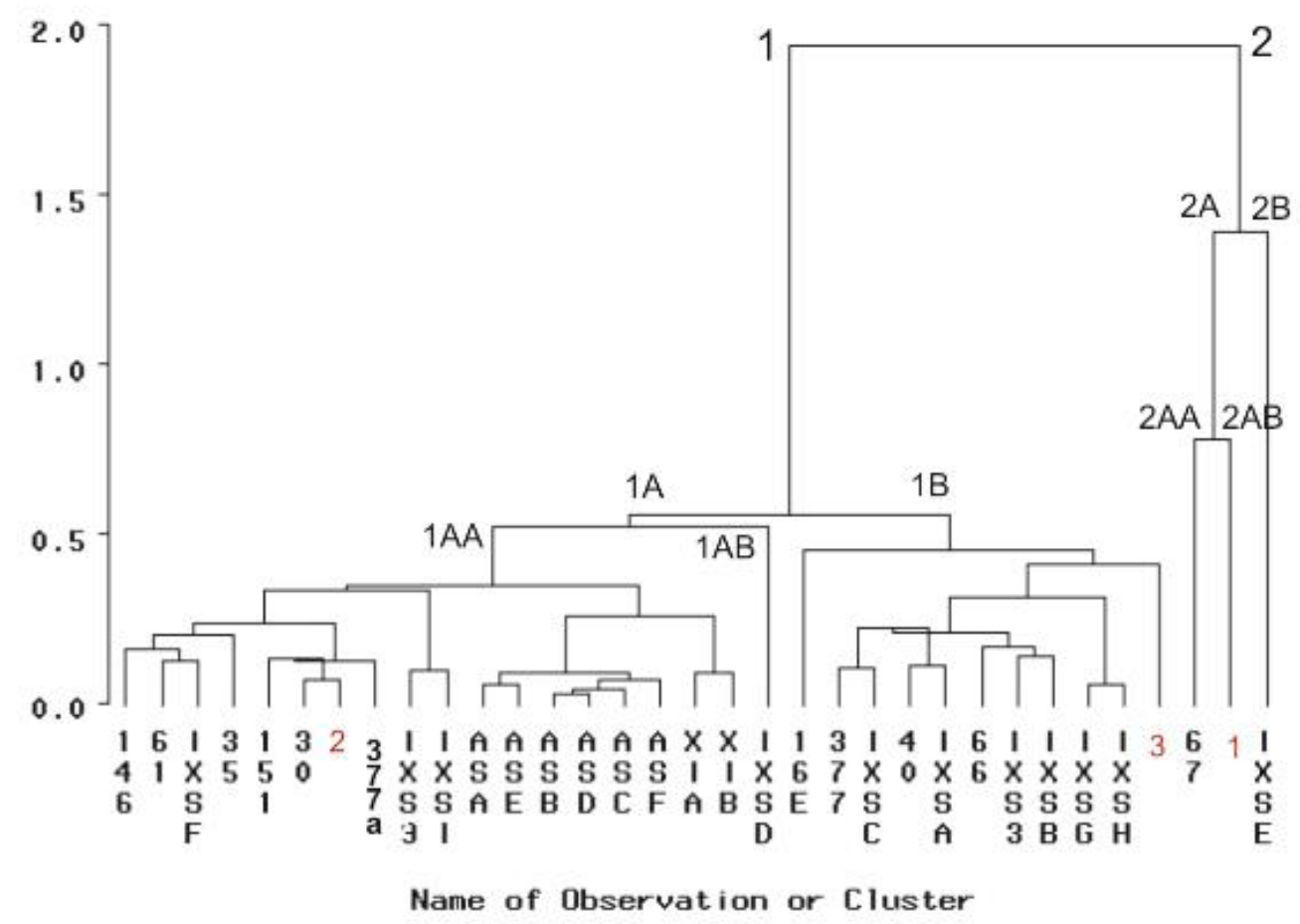

Figura 3 - Dendrograma com as 30 amostras de cerâmicas e 3 amostras de argilas do norte do Paraná (números em vermelho), analisadas pelo método de agrupamento médio (Extraída de CUNHA e SILVA et al., 2005). Extraída de CUNHA e SILVA et al., 2005.

\section{COMENTÁRIOS FINAIS}

Diferentes combinações de metodologias nuclear-atômico-moleculares têm sido empregadas no Laboratório de Física Nuclear Aplicada (LFNA) e em colaboração com outras instituições, para aplicações em muitas áreas, em especial, dentre elas a Arqueometria e Arte desde o início dos anos 90 (www.fisica.uel.br/gfna). Metodologias como a Espectrometria, Transmissão e Espalhamento de Raios Gama, Espectroscopia Mössbauer, Microscopia Eletrônica de Varredura e Transmissão, Microtomografia com Raios X, Fluorescência de raios X (EDXRF, PXRF, TXRF), Espectroscopia Raman, Radiografia Digital e Difração de Raios X, dentre outras, têm sido empregadas. Cada uma delas pode ser utilizada isoladamente ou combinadas, a fim de aumentar e aprofundar a qualidade da análise. Neste trabalho revisamos alguns trabalhos com cerâmicas arqueológicas empregando a Fluorescência de Raios X, a Espectroscopia Raman, a Transmissão de Raios Gama e a Microtomografia com Raios X apresentando os resultados de interesse para a análise destes objetos do patrimônio cultural. 


\section{REFERÊNCIAS}

APPOLONI, C. R., PARREIRA, P.S. and DE SOUZA, E. A gamma ray study of indian ceramics from southern Brazil. Conference Records of the 4th International Conference on Non-Destructive Testing of Works of Art, Berlin, V. 01, pp. 400-407, 1994.

APPOLONI, C. R. Non-destrutive analysis of brazilian archaeological pottery from the region of Londrina city. Conference Records of the 5th International Conference on Non-Destructive Testing and Environmental Evaluation for Study of Works of Art, V. 01, pp. 75-88, 1996.

APPOLONI, C. R., PARREIRA, P. S., SOUZA, E. de, QUACCHIA, J.C.A., NASCIMENTO FILHO, V.F. do, GIGANTE, G. E., CESAREO, R. e da CUNHA e SILVA, R. M. Estudo de cerâmicas arqueológicas do Paraná por técnicas nucleares não-destrutivas. Revista do Museu de Arqueologia e Etnologia da Universidade de São Paulo, São Paulo, V. 2, p. 135-149, 1997.

APPOLONI, C. R.; BLONSKI, Maria Sélia ; PARREIRA, P. S. ; SOUZA, Luiz A C . Study of the pigments elementary chemical composition of a painting in process of attribution to Gainsborough employing a portable X-rays fluorescence system. Nuclear Instruments \& Methods in Physics Research A, v. 580, p. 710-713, 2007.

APPOLONI, C. R., LOPES, F., MELQUIADES, F. L. e PARELLADA, C. I. In situ pigments study of rock art at Jaguariaíva 1 archaeological site (Paraná, Brazil) by portable energy dispersive X-ray fluorescence. FUMDHAMentos, V. 09, p. $555-562,2010$

APPOLONI, C. R. Recent developments in atomic/nuclear methodologies used for the study of cultural heritage objects. XXXV Brazilian Workshop on Nuclear Physics. AIP Conference Proceedings, Vol. 1529, p. 30-39, 2013.

APPOLONI, C. R., PARREIRA, P. S. e LOPES, F. Nineteen years of activities on Art, Archaeometry and Cultural Heritage Conservation at the Applied Nuclear Physics Laboratory (LFNA) UEL Brazil. 4.o Simposio Latinoamericano de Métodos de Física y Quimica en Arqueologia, Arte y Conservación de Patrimonio Cultural - LASMAC 2013, Bogotá. DVD Memorias Simposio LASMAC2013. Bogotá: Asociación Quimica Colombiana. Volume. único p. 1-5, 2013a.

APPOLONI, Carlos R.; LOPES, Fabio ; BRUNO, M. A. . Analysis of the painting 'Moema' by PXRF, TXRF and Raman Spectroscopy. In: BARBOSA, Karen (Org.). Moema - restauração / restoration. 1ed.São Paulo: Comunique Editorial, v. único, p. 59-78, 2013b.

APPOLONI, C.R., PARELLADA, C.I., JUSSIANI, E.I., MELQUIADES, F.L. and DE OLIVEIRA, F C P . Rock art paintings of Chapadinha shelters, Paraná state, Brazil - an in situ Raman study. Proceedings of the XInternational Symposium on RockArt - V Meeting The Brazilian Association of RockArt (ABAR), Teresina, p. 18, 2014.

BLONSKI, M. S. and APPOLONI, C. R. Pigments analysis and gold layer thickness evaluation of polychromy on wood objects by PXRF. Applied Radiation and Isotopes, V. 89, p. 47-52, 2014.

CUNHA e SILVA, R. M. da, NASCIMENTO FILHO, V.F., APPOLONI, C. R. e PEREZ, C.A. Fluorescência de Raios X aplicada a amostras arqueológicas. Revista do Museu de Arqueologia e Etnologia, São Paulo, V. 15/16, p. 371-382, 2005.

CURADO, J. F., ADDED, N. e RIZZUTTO, M. A. Evaluating the presence of porosity in Brazilian archaeological pottery associating X-radiography and PIXE. XXXV Brazilian Workshop on Nuclear Physics. AIP Conference Proceedings, Vol. 1529, p. 45-48, 2013. 
FERRARO, J., NAKAMOTO, K. and BROWN, C. Introductory Raman Spectroscopy. 2. ed., Elsevier, 434 p., 2003.

GALVÃO, T. D., LOPES, F. and APPOLONI, C. R. Exame da sensibilidade analítica em sistemas PXRF para análises de obsidianas. IN: BERTOLINO, S., CATTÁNEO, R. e IZETA, A. D. (org.) La arqueometría en Argentina y Latinoamérica, $1^{\text {a }}$ ed. Córdoba- Argentina, Universidad Nacional de Córdoba - Editorial de la Facultad de Filosofía y Humanidades, pp. 183-188, 2010.

IKEOKA, R. A. ; APPOLONI, C R ; MARCORI, O. ; Bandeira, Arkley M. . X-RAY FLUORESCENCE, RAMAN AND MULTIVARIATE STATISTICS ANALYSIS OF ARCHAEOLOGICAL POTTERY FROM SÃO LUIZ, MA, BRAZIL. In: 65th Annual Denver X-ray Conference, 2017, Rosemont. 2016 Denver X-ray Conference Proceedings - Advances in X-ray Analysis. Philadelphia: International Centre for Diffraction Data, 2017. v. 60. p. 190-199.

IKEOKA, R. A., APPOLONI, C. R., PARREIRA, P. S., FABIO, L. and BANDEIRA, A. M. PXRF and multivariate statistics analysis of pre-colonial pottery from northeast of Brazil. X-Ray Spectrometry, V. 41, p. 12-15, 2012.

IKEOKA, R. A. Análise de cerâmicas de quatro sítios arqueológicos (MA e SP) por seis métodos analíticos atômicos, moleculares e nuclear, 175 páginas. Tese. Doutorado em Física. Departamento de Física. Orientador Carlos R. Appoloni. Universidade Estadual de Londrina, Londrina, 17/12/2014.

JUSSIANI, Eduardo I. ; APPOLONI, C.R. ; TICONA, J. L. ; CRIALES, J. V. ; SAGARNAGA, J. ; SORIA, J. L. P. Análises morfológicas de cerâmicas de povoados de tiwanaku por microtomografia de raios x. In: VI simposio latinoamericano de física y química en arqueología, arte y conservación del patrimonio cultural - lasmac 2017, 2017, La Paz. Resumenes LASMAC 2017. La Paz: LASMAC 2017, v. único. p. 2-2, 2017.

KULKOVA, M. A. and KULKOV, A. M. Investigations of Early Neolithic ceramics from Eastern Europe by X -Ray microtomography and petrography. 2014 Annual Bruker microCT User Meeting, Ostend, Belgian, Eletronic Proceedings at http://www.skyscan.be/company/usersmeeting2014a.htm, 7 p., 2014.

MILHEIRA, R.G., APPOLONI, C.R. e PARREIRA, P.S. Arqueometria em cerâmicas Guarani no sul do Brasil: um estudo de caso. Revista do Museu de Arqueologia e Etnologia, São Paulo, 19: 355-364, 2009.

MiLler JR., T. O. Tecnologia cerâmica dos Caingang Paulistas. Arquivos do Museu Paranense - Nova Série, Etnologia, 2 , 51 p., 1978

Olivares, M., ZUluagA, M. C., OrTegA, L. A., MurelagA, X., AlOnso-OlaZABAl, A., URTEAGA, M., AMUNDARAY, L., ALONSO-MARTIN, I. and ETXEBARRIA, N. Characterization of fine wall and eggshell Roman pottery by Raman spectroscopy. Journal of Raman Spectroscopy, v. 41, p. 1543-1549, 2010.

PARREIRA, P. S., APPOLONI, C. R., VIEIRA, R. M. L., SCORZELLI, R. B., LE CORRE, L. and GUERRA, M. F. Precious metals determination in ancient coins by Portable ED-XRF Spectroscopy with a 238Pu source. ArcheoScience, revue d'archéométrie, V. 33, p. 313-318, 2009.

RASKOVSKA, A., MINCEVA-SUKAROVA, B., GRUPCE, O. and COLOMBAN, P. H. Characterization of pottery from Republic of Macedonia II. Raman and infrared analyses of glazed pottery finds from Skopsko Kale. Journal of Raman Spectroscopy, v. 41, p. 431-439, 2010.

RAVAUD, Élisabeth ; SOUZA, Luiz ; LEÃO, Alexandre ; ROSADO, Alessandra ; APPOLONI, Carlos R. ; LOPES, Fabio ; PARREIRA, Paulo Sergio . Hymeneus travestido assitindo a uma dança em honra a Príapo: estudo científico. In: Pierre Curie. (Org.). Poussin - Restauração - Hymeneus travestido assitindo a uma dança em honra a Príapo. São Paulo: Imprensa Oficial, 2009, v. único, p. 81-92. 
RIZZO, M. de M., APPOLONI, C. R. e PARREIRA, P. S. Remoção de sais insolúveis sobre pinturas murais à base de água com acompanhamento in situ por equipamento portátil de EDXRF. Anais do lo Simpósio LatinoAmericano sobre Métodos Físicos e Químicos em Arqueologia, Arte e Conservação de Patrimônio Cultural. TecArt, São Paulo, CRROM, p. 239-247, 2007.

SILVA, F.A., APPOLONI, C. R., QUIÑONES, F.R.E., SANTOS, A.O., SILVA, L.M. da, BARBIERI, P.F. e NASCIMENTO FILHO, V. F. do. A Arqueometria e a Análise de Artefatos Cerâmicos: um estudo de fragmentos cerâmicos etnográficos e arqueológicos por fluorescência de Raios X (EDXRF) e transmissão Gama. Revista de Arqueologia (Belém), V. 17, p. 41-61, 2004.

VAN GRIEKEN, R. E. and MARKOWICZ, A. A. Handbook of X-Ray Spectrometry, Marcel Dekker Inc., New York, pp. 199-236, 2002.

Recebido em: 30/01/2018

Aprovado em: 20/03/2018

Publicado em: 30/11/2018 